Rapid Communication

\title{
On the Repulsive Interaction Between Strongly Overlapping Double Layers of Charge-regulated Surfaces ${ }^{\text {is }}$
}

\author{
A.P. Philipse ${ }^{a, *}$, R. Tuinier ${ }^{a, b}$, B.W.M. Kuipers ${ }^{a}$, A. Vrij ${ }^{a}$, M. Vis ${ }^{b, *}$ \\ a Van 't Hoff Laboratory for Physical and Colloid Chemistry, Debye Institute for Nanomaterials Science, Utrecht University, Padualaan 8, Utrecht 3584 CH, The \\ Netherlands \\ b Laboratory of Physical Chemistry, Department of Chemical Engineering and Chemistry \& Institute for Complex Molecular Systems, Eindhoven University of Technology, \\ PO Box 513, Eindhoven 5600 MB, The Netherlands
}

\begin{abstract}
A B S T R A C T
The Donnan equilibrium is employed to evaluate the entropic repulsion between two charged plates that feature charge regulation and are in equilibrium with a reservoir solution of monovalent salt. This approach represents the zero-field limit of the Poisson-Boltzmann equation, valid for strongly overlapping electrical double layers. We show that this scenario features an intrinsic length scale, which serves as the unscreened pendant of the Debye length for strongly overlapping double layers. In general, the scaling of the disjoining pressure with interplate distance is dependent on the boundary conditions (constant charge, constant potential, or charge regulation). Surprisingly, here we find for sufficiently low potentials the same inverse-square decay as for constant charge surfaces. We test the validity of the zero-field limit by comparison with self-consistent field lattice computations that invoke the full Poisson equation for finitely sized ions between two charge-regulated plates.
\end{abstract}

The electrical double-layer repulsion between two charged surfaces in equilibrium with a salt reservoir is conventionally [1-10] evaluated under the assumption that surfaces are sufficiently far apart such that their double-layers only weakly interact. This so-called 'weak-overlap approximation' [4] implies that the electrical potential in the mid-plane between the two surfaces is small (though surface potentials, nevertheless, may be high). Within this weak-overlap approximation, the Poisson-Boltzmann (PB) equation eventually yields osmotic disjoining pressures that decay exponentially; the typical decay length being the Debye screening length $\kappa^{-1}$ which measures the thickness of a diffuse electrical double-layer in solution. Together with Van der Waals attractions, one arrives at the exponentially screened, classical DLVO potential [1-4], which is applicable to dilute colloidal fluids in which the average colloid-colloid distance is (much) larger than $\kappa^{-1}$.

When distances between charged surfaces are comparable to or less than $\kappa^{-1}$ such that double-layers strongly overlap and the weak-overlap approximation breaks down, one must resort to more complicated solutions containing elliptic functions [11] or to numerical solutions. For low surface potentials with plates in close proximity and in presence of background salt, analytical approximations exist featuring a peculiar inverse square decay of the disjoining pressure with the inter-plate separation [11]. To the best of our knowledge, however, these approximations are only known for the boundary condition of constant surface charge [11-13], but not for surfaces featuring charge regulation, even though these boundary conditions in general do not lead to the same scaling behavior of the disjoining pressure [14]. It should be mentioned that an inverse square decay is also known for the salt-free (counter-ion only) limit at large separations, even in case of charge regulation $[11,15]$.

Here we demonstrate a relatively straightforward and analytical treatment of the disjoining pressure between strongly overlapping flat double-layers featuring charge regulation on the basis of the Donnan equilibrium. This method exploits the circumstance that, on approach of two charged plates, absolute values of the potential in the electrolyte solution between the plates rise, but potential gradients on average become smaller. Thus to analyze repulsions between plates at a distance comparable to the Debye length, the limit of a weak electric field has to be considered [13] rather than the limit of a high potential [1,2]. From the weak-field point of view, the simplest starting point is obviously the case where the electric field is zero everywhere, i.e., the ions in the inter-plate electrolyte solution are homogeneously distributed in a constant electrical potential, also known as the Donnan potential $[16,17]$.

Zero-field disjoining pressures as function of the Donnan potential

\footnotetext{
Acknowledgements: M.V. and R.T. would like to thank Prof. F.A.M. Leermakers for his help and providing the SFbox software package, which incorporated surprising features that sparked fruitful discussions.

" Corresponding authors.

E-mail addresses: A.P.Philipse@uu.nl (A.P. Philipse), M.Vis@tue.nl (M. Vis).
} 
are derived as follows. Consider two parallel plates separated by an electrolyte solution $S$ in thermodynamic equilibrium with a large reservoir containing a constant number density $\rho_{s}$ of salt molecules of fully dissociated 1-1 electrolyte. The electrical potential in S, relative to the potential in the reservoir, equals the constant Donnan potential $\bar{\Psi}$, where the bar indicates the zero-field assumption. The ions in $S$ are homogeneously distributed in zero electric field, with average densities given by the Boltzmann distributions for ideal ions:

$\bar{\rho}_{ \pm}=\rho_{\mathrm{s}} \exp (\mp \bar{u}), \quad$ where $\bar{u} \equiv \frac{e \bar{\Psi}}{k_{\mathrm{B} T}}$.

Here $\bar{u}$ represents the dimensionless Donnan potential, $e$ is the elementary charge, and $k_{\mathrm{B}} T$ is the thermal energy. The excess ion density $\Delta \rho$ in $\mathrm{S}$ relative to the ion density in the reservoir is $\Delta \rho \equiv \bar{\rho}_{+}+\bar{\rho}-2 \rho_{\mathrm{s}}$, which can be combined with Eq. (1) to yield $\Delta \rho=2 \rho_{\mathrm{s}}(\cosh \bar{u}-1)$. For ideal ions obeying Van 't Hoff's law, the disjoining pressure equals $\Delta \Pi_{\mathrm{d}}=\Delta \rho k_{\mathrm{B}} T$, which in terms of the Donnan potential reads

$\frac{\Delta \Pi_{\mathrm{d}}}{2 \rho_{\mathrm{s}} k_{\mathrm{B}} T}=\cosh \bar{u}-1$.

Here pressures are scaled on the osmotic pressure $2 \rho_{\mathrm{s}} k_{\mathrm{B}} T$ of the salt reservoir. Since $\cosh \bar{u}>1$, the ion density in $\mathrm{S}$ always exceeds the reservoir ion density so charged plates always spontaneously separate ('disjoin'). Eq. (2) is exact for homogeneously distributed, ideal ions, and is independent of the extent of surface charge regulation. The interplate Donnan potential $\bar{u}$ in Eq. (2) follows from the charge density on the surfaces and the electro-neutrality condition, which we will consider next.

Suppose the surfaces have a number of sites per unit area $\sigma_{\text {tot }}$ that each may release a mono-valent positive counter-ion into solution; a practical example, addressed later, are the protons released by the dissociating hydroxyl groups from a silica surface. When $\sigma$ denotes the density of dissociated, negatively charged sites and $\bar{\rho}_{+}$the density of counter-ions in $S$, the dissociation equilibrium constant is given by $K=\left(\sigma \bar{\rho}_{+}\right) /\left(\sigma_{\text {tot }}-\sigma\right)$. On substitution of the cation Boltzmann distribution from Eq. (1) the degree of dissociation $\sigma / \sigma_{\text {tot }}$ follows as

$\frac{\sigma}{\sigma_{\text {tot }}}=\frac{K}{K+\bar{\rho}_{+}}=\frac{k}{k+\exp (-\bar{u})}$,

where we introduced the dimensionless equilibrium constant

$k \equiv K / \rho_{\mathrm{s}}$.

When the plates move apart, the charge density $\sigma$ increases according to Eq. (3) and approaches the maximum value

$\sigma_{\max }=\sigma_{\text {tot }} \frac{k}{1+k}$,

achieved for the case of zero Donnan potential for a single free plate.

Next we employ the electro-neutrality condition $\bar{\rho}_{+} h=\bar{\rho}-h+2 \sigma$, for two negatively charged plates at inter-plate distance $h$, to find on substitution of $\sigma$ from Eq. (3):

$\frac{\bar{\rho}_{+}-\bar{\rho}}{2 \rho_{\mathrm{s}}}=\frac{1+k}{k+\exp (-\bar{u})} \frac{\lambda}{h}$.

Here we have introduced a characteristic length $\lambda$ defined as

$\lambda \equiv \frac{k}{1+k} \frac{\sigma_{\mathrm{tot}}}{\rho_{\mathrm{s}}}=\frac{\sigma_{\max }}{\rho_{\mathrm{s}}}$.

The length scale $\lambda$, incidentally, is a distinguishing feature of the zerofield Donnan limit, being the unscreened pendant of the Debye length

$\kappa^{-1}=\frac{1}{\sqrt{8 \pi \rho_{\mathrm{s}} \ell_{\mathrm{B}}}}$,

(where $\ell_{B}$ is the Bjerrum length), which is the length scale that appears
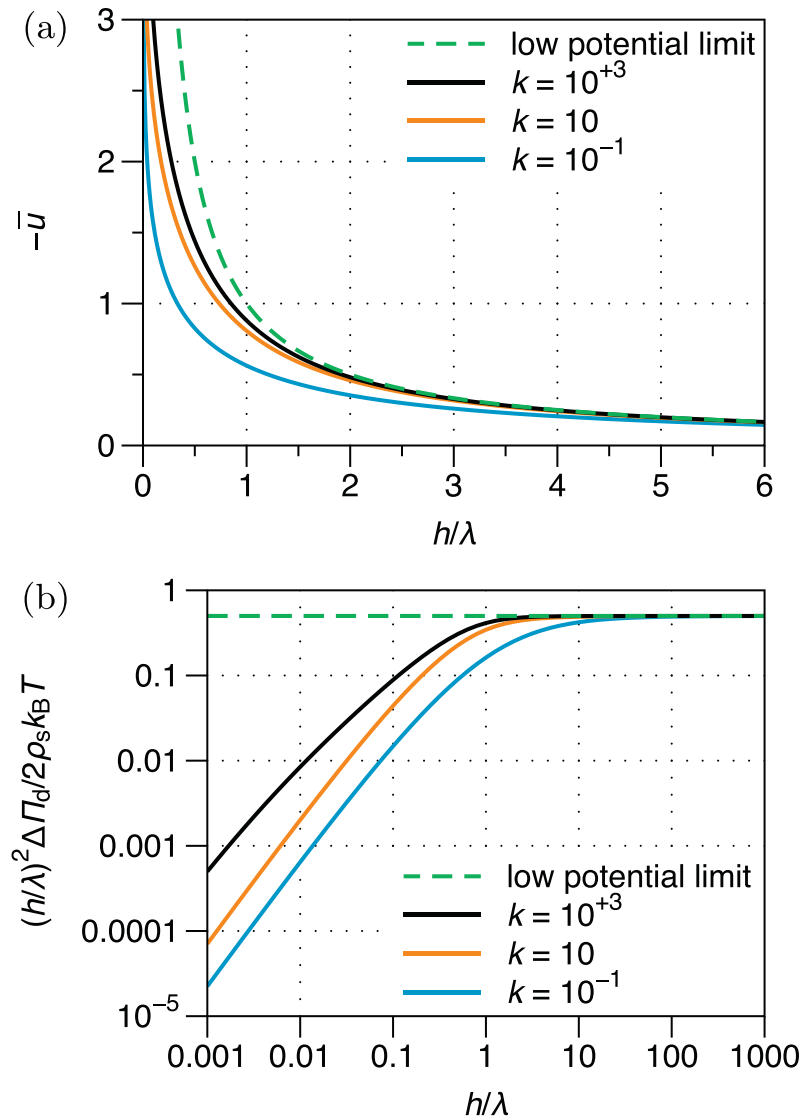

Fig. 1. (a) Zero-field Donnan potential $\bar{u}$ and (b) disjoining pressures $\Delta \Pi_{\mathrm{d}}$ versus the dimensionless inter-plate distance $h / \lambda$, with $\lambda$ defined in Eq. (7). The potential follows from Eq. (10) and the disjoining pressure through subsequent application of Eq. (2). The green dashed lines indicate the low-potential, large separation limits of Eqs. (12) and (15). Increasing the dimensionless dissociation constant $k$ increases the magnitude of $\bar{u}$ for a given inter-plate distance. Both potential and disjoining pressure show universal behavior at large separations. (For interpretation of the references to color in this figure legend, the reader is referred to the web version of this article.)

in the exponentially screened repulsions between plates with weak double-layer overlap [1-13]. Whereas $\kappa^{-1}$ indicates the typical distance over which screening occurs, its pendant $\lambda$ indicates the inter-plate distance $h \approx \lambda$ at which counter-ions and background salt have a comparable effect on the Donnan potential: for $h \ll \lambda$ counter-ions dominate, while for $h \gg \lambda$ background salt overshadows the contribution of the counter-ions. Alternatively, $\lambda$ can be expressed in terms of the Gouy-Chapman length $\ell_{\mathrm{GC}}=\left(2 \pi \ell_{\mathrm{B}} \sigma_{\mathrm{tot}}\right)^{-1}$ of a fully dissociated plate as

$\lambda=4 \frac{k}{k+1} \ell_{\mathrm{GC}}^{-1} \kappa^{-2}$.

We return to Eq. (6), which on substitution of the Boltzmann distribution, Eq. (1), yields an expression relating potential $\bar{u}$ and interplate distance $h$ :

$\frac{k+\exp (-\bar{u})}{1+k} \sinh \bar{u}=-\frac{\lambda}{h}$.

This equation is cubic in $\exp (-\bar{u})$. Fig. 1a illustrates that for given inter-plate distance $h$ the absolute value of the zero-field potential in Eq. (10) rises upon increasing the dimensionless dissociation constant $k$, an increase which corresponds with enhanced surface charge density on the plates, see Eq. (3). If for given $k$ the plates move apart, the Donnan potential decreases and, consequently, the disjoining pressure decays.

To find the leading term in the pressure decay we first expand exponents $\exp ( \pm \bar{u})$ in Eq. (10) up to order $\bar{u}^{2}$, to find the solution 
$\bar{u} \simeq \frac{1}{2}(1+k)\left[1-\sqrt{1+\frac{4 \lambda}{(1+k) h}}\right]$

$\simeq-\frac{\lambda}{h} \quad$ for $|\bar{u}| \ll 1$.

Substitution of this Donnan potential in an expansion of Eq. (2) up to order $\bar{u}^{2}$ then yields the following disjoining pressure:

$\frac{\Delta \Pi_{\mathrm{d}}}{2 \rho_{\mathrm{s}} k_{\mathrm{B}} T} \simeq \frac{1}{2} \bar{u}^{2}$

$\simeq \frac{1}{2}\left(\frac{\lambda}{h}\right)^{2}\left(1-\frac{2}{1+k} \frac{\lambda}{h}+\cdots\right)$

$\simeq \frac{1}{2}\left(\frac{\lambda}{h}\right)^{2} \quad$ for $|\bar{u}| \ll 1$.

Thus, disjoining pressures harbor at small inter-plate potentials, i.e., at large inter-plate distances, an inverse-square decay $(h / \lambda)^{-2}$. This decay is independent of the extent of charge regulation of the two surfaces and becomes identical to that of constant charge surfaces [11-13] for $k \rightarrow \infty$. In other words, disjoining pressures merge into one universal decay when inter-plate distances are scaled on $\lambda$, see also Fig. 1b. Already in the first additional term of order $(h / \lambda)^{-3}$ in Eq. (14) this 'universality' disappears due to a term inversely proportional to $1+k$. When plates move even closer together such that Donnan potentials significantly rise, the $h$-dependence of the disjoining pressure itself depends on the dissociation constant $k$. This $k$-dependence, incidentally, is due to salt expulsion by the charged plates to the reservoir: on approaching contact, the $k$-dependent counter-ion density in the inter-plate space $S$ dominates the disjoining pressure. The decay from Eq. (15) occurs in the opposite regime, where salt molecules in S in turn overshadow the counter-ions.

The inverse-square decay from Eq. (15) manifests itself only when the maximum charge density $\sigma_{\max }$ on free, non-interacting plates is sufficiently low. Inter-plate distances $h$ must be large enough to attain a small Donnan potential $\bar{u}$ but, on the other hand, should also satisfy $h \lesssim$ $\kappa^{-1}$ to be in the unscreened, zero-field region in the first place. The Donnan potential is small for inter-plate distances $h \gtrsim \lambda$, so the inversesquare decay falls inside a window of inter-plate distances approximately given by $\lambda \lesssim h \lesssim \kappa^{-1}$. For this window to exist, the maximum charge density on single plates must be below a threshold maximum density of dissociated groups that occurs when $\lambda$ in Eq. (7) equals the Debye length:

$\sigma_{\max }^{\text {thr }} \equiv \rho_{\mathrm{s}} \kappa^{-1}$.

Some numerical examples for silica surfaces in water are listed in Table 1. Due to their weakly acidic surface hydroxyl groups, silica surfaces in close proximity display surface charge regulation $[18,19]$. According to Ref. [19] smooth, non-porous silica surfaces have silanol densities of about $\sigma_{\text {tot }}=5 \mathrm{~nm}^{-2}$. For the dissociation equilibrium $\equiv \mathrm{SiOH} \rightleftharpoons \equiv \mathrm{SiO}^{-}+\mathrm{H}^{+}$of surface silanol groups, $K \approx 10^{-7} \mathrm{M}$ (i.e., $\left.\mathrm{p} K_{\mathrm{a}}=7\right)$ [19]. These values for $\sigma_{\text {tot }}$ and $K$ lead to the charge densities in Table 1, for various concentrations $\rho_{\mathrm{s}}$ of a simple strong acid (e.g., $\mathrm{HCl}$ or $\mathrm{HNO}_{3}$ ) as the only salt. Note that in Table $1 k \equiv K / \rho_{\mathrm{s}} \ll 1$ : silica surfaces in water are always weakly dissociated; the highest fraction of dissociated silanol groups in Table 1 , achieved for $\rho_{s}=10^{-5} \mathrm{M}$, is only $1 \%$. The requirement $\sigma_{\max } \lesssim \sigma_{\max }^{\text {thr }}$ for having a window for the inversesquare decay is fulfilled for $\mathrm{pH} 2$ and 3 , see Table 1 . Note that for $\rho_{\mathrm{s}}=10^{-3} \mathrm{M}$, the predicted window for an algebraic decay is fairly broad, from $\lambda \approx 0.8 \mathrm{~nm}$ to $1 / \kappa \approx 10 \mathrm{~nm}$. It should be mentioned that, in practice, silica at $\mathrm{pH}$ below 3 starts to acquire positive surface charges due to other equilibria [20]; therefore we do not further consider $\mathrm{pH}<3$ here.

To quantitatively test the Donnan theory, we performed self-consistent field (SCF) calculations of the thermodynamic properties of two parallel charge-regulated plates in an electrolyte solution, see Figs. 2-4.
Table 1

Parameters for silica surfaces in simple acid ${ }^{\mathrm{a}}$.

\begin{tabular}{lllllll}
\hline $\mathrm{pH}$ & $\rho_{\mathrm{s}}(\mathrm{M})^{\mathrm{b}}$ & $k^{\mathrm{c}}$ & $\sigma_{\max }\left(\mathrm{nm}^{-2}\right)^{\mathrm{d}}$ & $\sigma_{\max }^{\mathrm{thr}}\left(\mathrm{nm}^{-2}\right)^{\mathrm{e}}$ & $\lambda(\mathrm{nm})^{\mathrm{f}}$ & $\kappa^{-1}(\mathrm{~nm})^{\mathrm{g}}$ \\
\hline 2 & $10^{-2}$ & $10^{-5}$ & $5 \cdot 10^{-5}$ & $1.8 \cdot 10^{-2}$ & $8.3 \cdot 10^{-3}$ & 3.0 \\
3 & $10^{-3}$ & $10^{-4}$ & $5 \cdot 10^{-4}$ & $6.0 \cdot 10^{-3}$ & $8.3 \cdot 10^{-1}$ & 9.6 \\
4 & $10^{-4}$ & $10^{-3}$ & $5 \cdot 10^{-3}$ & $1.8 \cdot 10^{-3}$ & $8.3 \cdot 10^{+1}$ & 30 \\
5 & $10^{-5}$ & $10^{-2}$ & $5 \cdot 10^{-2}$ & $6.0 \cdot 10^{-4}$ & $8.2 \cdot 10^{+3}$ & 96
\end{tabular}

a The silica surfaces have a total number density $\sigma_{\text {tot }}=5 \mathrm{~nm}^{-2}$ of dissociable surface silanol groups with dissociation constant $K=10^{-7} \mathrm{M}=60 \mu \mathrm{m}^{-3}$ [19].

${ }^{\mathrm{b}}$ Constant concentration of 1-1 strongly acidic electrolyte (e.g., $\mathrm{HCl}$ or $\mathrm{HNO}_{3}$ ) in a salt reservoir.

${ }^{\mathrm{c}} k=K / \rho_{\mathrm{s}}$; dimensionless dissociation constant, see Eq. (4).

${ }^{\mathrm{d}} \sigma_{\text {max }}=k \sigma_{\text {tot }} /(1+k)$; number density of dissociated silanol groups on a single, free surface, see Eq. (5).

e $\sigma_{\max }^{\text {thr }}=\rho_{\mathrm{s}} \kappa^{-1}$; threshold charge number density for a window of inverse-square decay to exist, see Eq. (16).

${ }^{\mathrm{f}}$ Minimum inter-plate separation to access region of small Donnan potential.

${ }^{\mathrm{g}}$ Debye screening length from Eq. (8), which is approximately the maximum interplate separation to stay within unscreened regime.

In contrast to Donnan theory for point ions, the SCF computations solve the full Poisson equation (on a mean-field level) for finitely sized ions and solvent molecules, incorporating also the effects of, e.g., the Stern layer. SCF theory has been extensively discussed elsewhere [21-25]. The calculations yield, e.g., electrostatic potential and concentration profiles, chemical potentials, and the free energy of the system. The mid-plane potential (where the field is zero) of the computations is compared to the zero-field Donnan potential. The disjoining pressure follows from the derivative of the semi-open grand potential with respect to inter-plate distance. The input of the SCF calculations is as follows. The size of a lattice site is set to $b=0.3 \mathrm{~nm}$. All $\chi$ parameters are set to zero (athermal Flory-Huggins interactions) and the relative permittivity of the solvent equals 80 to model water. Water self-dissociation is modeled with an equilibrium constant $\mathrm{p} K_{\mathrm{w}}=14$, leading to a minimum ionic strength of $10^{-7} \mathrm{M}$ at $\mathrm{pH} 7$ which increases at different $\mathrm{pH}$. The reported ionic strength is the total in the reservoir due to any added inert background salt and the acid-base equilibrium at given $\mathrm{pH}$. The two plates are grafted with weakly acidic sites located directly next to the plates with a density $\sigma_{\text {tot }}$. The minimum number of lattice layers between the plates is therefore 2 , such that the grafted groups touch but do not interpenetrate.

For Fig. 2a the parameters were chosen to model silica surfaces with weakly acidic silanol groups $\left(\sigma_{\text {tot }}=5 \mathrm{~nm}^{-2}, \mathrm{p} K_{\mathrm{a}}=7\right.$, and no additional salt) at $\mathrm{pH} 5,4$, and 3 . Under these conditions, we find quantitative agreement between Donnan theory and the SCF computations. Only a slight deviation is observed at small separations, owing to the finite volume of dissociating surface groups in the SCF calculations, disregarded in the Donnan theory. At pH 3, the low potential limits of Eqs. (12) and (15) are reached (green dashed curves).

For the situation in Fig. 2a, the ionic strength can only be adapted by changing the $\mathrm{pH}$ as there is no additional background salt. To separate the effects of changing $\mathrm{pH}$ and ionic strength, SCF calculations were also performed with additional background salt at fixed $\mathrm{pH}$. Although our Donnan theory does not explicitly include the effect of inert salt, it can still be used under these conditions by choosing effective values for $\mathrm{p} K_{\mathrm{a}}$ and $\mathrm{pH}$ while keeping their difference constant. These computations, with results shown in Fig. 2b, are performed for $\sigma_{\text {tot }}=1 \mathrm{~nm}^{-2}$ with $\mathrm{p} K_{\mathrm{a}}=7$, for $10^{-5}, 10^{-4}$, and $10^{-3} \mathrm{M}$ total salt at $\mathrm{pH}$ 7. Despite the lower total surface density $\sigma_{\text {tot }}$, the mid-plane potential is more negative due to a larger degree of dissociation and therefore the dimensionless disjoining pressure is higher. This also entails that in Fig. 2b the low potential limit is not reached. Additionally, the electric fields are larger, leading to greater deviations between the zero-field Donnan theory and SCF computations, especially for higher salt concentrations at larger inter-plate distances $h$. This is because the Donnan approach does not reproduce the exponential 

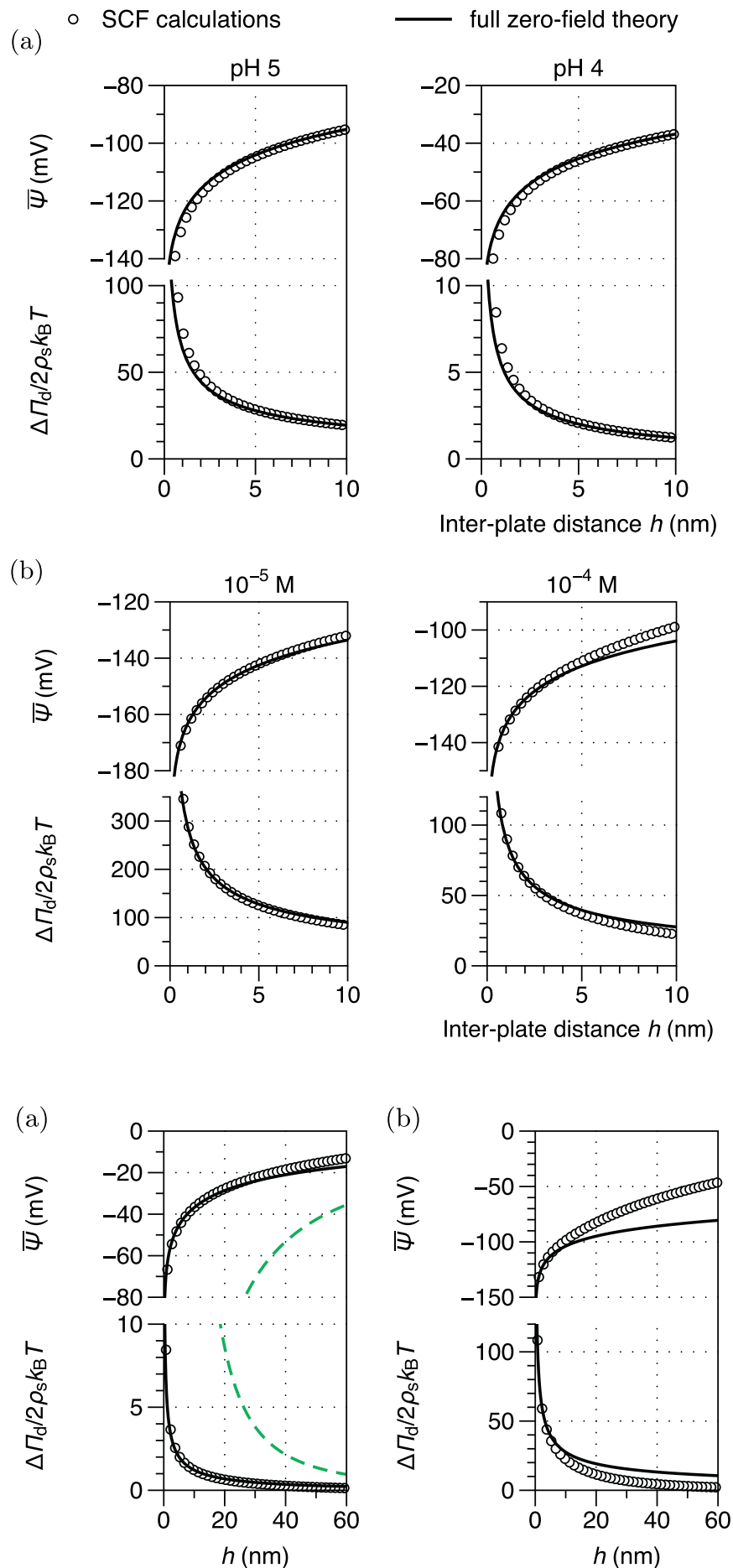

Fig. 3. Comparison between the zero-field approximation and self-consistent field calculations for larger inter-plate distances $h$. (a) Same as Fig. 2a, pH 4; (b) same as Fig. 2b, $10^{-4} \mathrm{M}$ salt.

decay due to screening at larger separations, as the zero-field limit by definition implies the absence of screening. However, the minor discrepancy at small $h$ between zero-field theory and SCF is reduced owing to the smaller value of $\sigma_{\text {tot }}$ and the correspondingly smaller volume occupied by the surface groups.

The situation at larger inter-plate distances of up to $60 \mathrm{~nm}$ is shown in Fig. 3 for two examples: $\mathrm{pH} 4$ with no additional background salt (Fig. 3a) and $\mathrm{pH} 7$ with $10^{-4} \mathrm{M}$ salt (Fig. 3b). Evidently Donnan theory breaks down at inter-plate distances comparable to the Debye length, here $\kappa^{-1}=30 \mathrm{~nm}$. However, for reasons mentioned previously, the
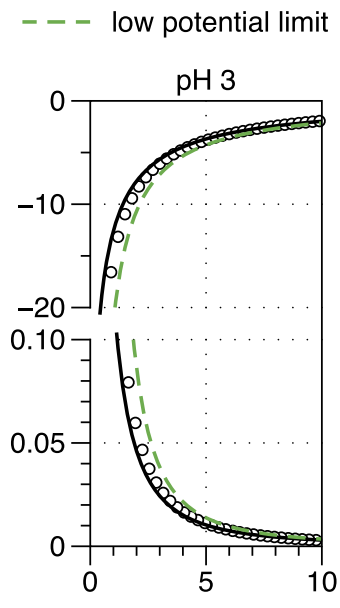

Fig. 2. Comparison between the zero-field approximation and numerical self-consistent field lattice calculations for the mid-plane potential $\bar{\Psi}$ and disjoining pressure $\Delta \Pi_{\mathrm{d}} /\left(2 \rho_{\mathrm{s}} k_{\mathrm{B}} T\right)$ of two charge-regulating plates in an electrolyte solution. Calculations (a) for $\sigma_{\text {tot }}=5 \mathrm{~nm}^{-2}$ at $\mathrm{pH} 5,4$, and 3 with $\mathrm{p} K_{\mathrm{a}}=7$, with no additional salt and (b) for $\sigma_{\text {tot }}=1 \mathrm{~nm}^{-2}$ at $\mathrm{pH} 7$ with $\mathrm{p} K_{\mathrm{a}}=7$, for $10^{-5}$, $10^{-4}$, and $10^{-3} \mathrm{M}$ total salt. The SCF calculations (open circles) are compared with full zero-field theory [solid black curves, Eqs. (2) and (10)], and low potential approximations [green dashed curves, Eqs. (12) and (15)]. (For interpretation of the references to color in this figure legend, the reader is referred to the web version of this article.)
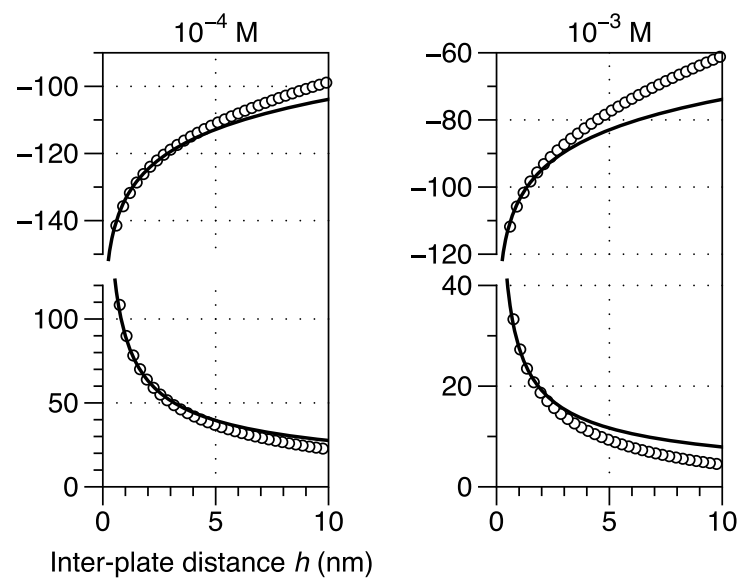

deviations are larger in Fig. 3b due to an enlarged degree of dissociation and more negative potentials.

Fig. 4a shows SCF calculations for two silica surfaces at low $\mathrm{pH}$ (Table 1) with charge densities below the threshold value from Eq. (16). The disjoining pressures between the silica surfaces at $\mathrm{pH} 3$ (from Fig. 2a) are plotted such that the inverse square decay manifests itself as a constant. Under the conditions of Fig. 4a, the minimum inter-plate distance required to enter the low-potential region is predicted, see Table 1 , to be about $1 \mathrm{~nm}$; the upper limit of the unscreened regime is given by $\kappa^{-1}$, implying a plateau for $1 \mathrm{~nm} \lesssim h \lesssim 10 \mathrm{~nm}$, which indeed can be discerned in Fig. 4a. The range of the plateau can be expanded by increasing $\kappa^{-1}$ or decreasing the minimum distance required to access the low-potential limit (e.g., increasing the $\mathrm{p} K_{\mathrm{a}}$ ). This is done in Fig. 4b, which shows calculations for parallel plates at $\mathrm{pH} 7$, with $\sigma_{\text {tot }}=1 \mathrm{~nm}^{-2}, \mathrm{p} K_{\mathrm{a}}=12$ and $10^{-4} \mathrm{M}$ total salt. The inverse square decay is now predicted to lie roughly in the range of $0.2-30 \mathrm{~nm}$, a prediction that is clearly confirmed by the SCF results in Fig. $4 \mathrm{~b}$, where the plateau is significantly broader than in Fig. 4a.

In conclusion, we have shown that the disjoining pressure between two charged surfaces with extensive double-layer overlap harbors a universal inverse-square decay $(h / \lambda)^{-2}$ in the zero-field Donnan limit for low potentials. This decay is independent of the extent of charge regulation, which is encompassed in the characteristic length $\lambda$. While in general the scaling behavior of the disjoining pressure depends on the boundary conditions [14], here we find surprisingly the same inverse square decay as for plates with a constant charge [11-13]. Predictions from the Donnan theory are quantitatively confirmed by numerical self-consistent field (SCF) lattice computations for inter-plate distances smaller than approximately the Debye length. For low Donnan potentials, the SCF calculations confirm the inverse-square 


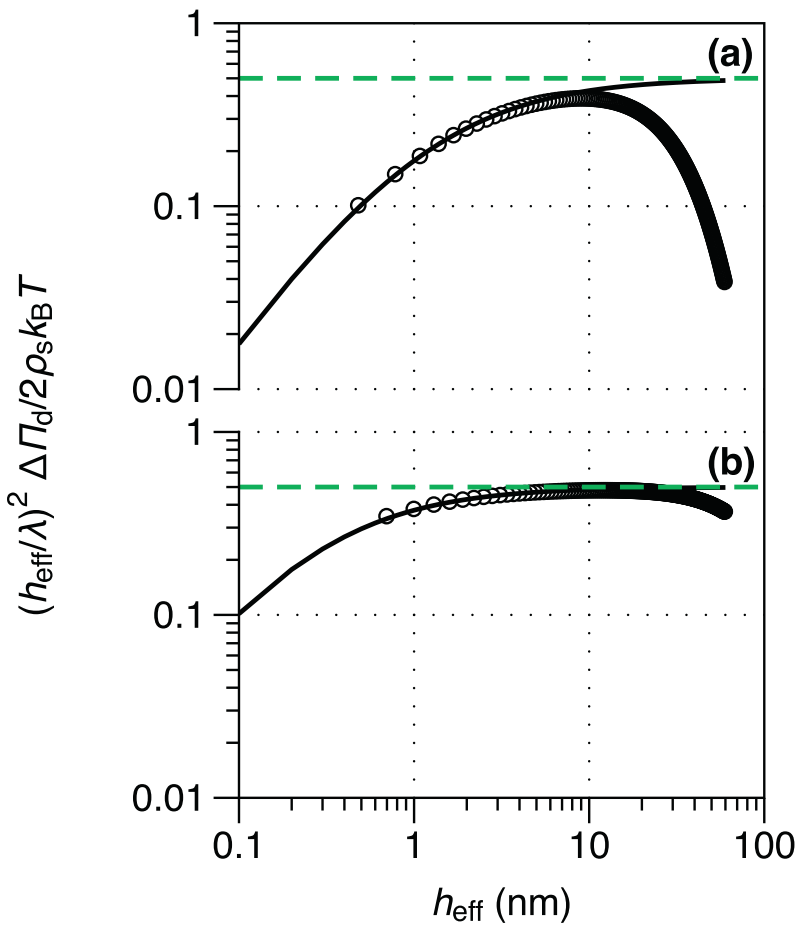

Fig. 4. Disjoining pressure versus inter-plate distance, plotted in such a way that the lowpotential inverse-square decay is visible as a horizontal plateau. The effective inter-plate distance $h_{\text {eff }}=h-2 \sigma_{\text {tot }} b^{3}$ accounts for the finite volume of the dissociating surface groups. The SCF computations (open circles) are for (a) two silica surfaces at $\mathrm{pH} 3$, with $\sigma_{\text {tot }}=5 \mathrm{~nm}^{-2}, \mathrm{p} K_{\mathrm{a}}=7$, and no additional background salt, and (b) for two surfaces at $\mathrm{pH} 7$, with $\sigma_{\text {tot }}=1 \mathrm{~nm}^{-2}, \mathrm{p} K_{\mathrm{a}}=12$ and $10^{-4} \mathrm{M}$ total salt. SCF results are compared to full zero-field theory [solid black curves, Eq. (2)] and low potential approximation [green dashed lines, Eq. (15)].

decay of the disjoining pressure. From a practical case study on weakly acidic silica surfaces in water we deduce-from both Donnan theory and SCF computations-that the surfaces exhibit charge-regulated zerofield repulsions for a wide electrolyte concentration range.

We have considered two parallel, infinite plates-an obvious choice of geometry to simplify calculations. In future work we seek for the transition from the osmotic disjoining pressure between flat plates, to the osmotic pressure of a dense dispersion of colloidal spheres where the average particle surface-to-surface distance is below the Debye length. We expect that the osmotic equation of state of such a concentrated dispersion comprises unscreened algebraically decaying particle interactions, instead of screened exponentials.

\section{References}

[1] E.J.W. Verwey, J.T.G. Overbeek, Theory of the Stability of Lyophobic Colloids, Dover Publications, 1999.

[2] B. Chu, P.J.W. Debye, Molecular Forces: Based on the Baker Lectures of Peter J.W. Debye, Interscience Publishers, 1967.

[3] J.T.G. Overbeek, Colloid Science, 2 Elsevier, Amsterdam, 1952.

[4] J.N. Israelachvili, Intermolecular and Surface Forces, Academic Press, 2015.

[5] T. Cosgrove, Colloid Science: Principles, Methods and Applications, John Wiley \& Sons, 2010.

[6] K. Dill, S. Bromberg, Molecular Driving Forces: Statistical Thermodynamics in Biology, Chemistry, Physics, and Nanoscience, Garland Science, 2010.

[7] D.F. Evans, H. Wennerström, The Colloidal Domain, Wiley-Vch, 1999.

[8] P.C. Hiemenz, R. Rajagopalan, Principles of Colloid and Surface Chemistry, Marcel Dekker, Mar. 1997.

[9] W.B. Russel, D.A. Saville, W.R. Schowalter, Colloidal Dispersions, Cambridge University Press, 1989.

[10] T.F. Tadros, Colloid Stability: The Role of Surface Forces, Wiley-Vch, 2007.

[11] T. Markovich, D. Andelman, R. Podgornik, Charged Membranes: Poisson-Boltzmann Theory, DLVO Paradigm and Beyond, (Mar. 2016) arxiv:1603.09451.

[12] P.M. Biesheuvel, Simplifications of the Poisson-Boltzmann equation for the electrostatic interaction of close hydrophilic surfaces in water, J. Colloid Interface Sci. 238 (2001) 362-370.

[13] A.P. Philipse, B.W.M. Kuipers, A. Vrij, Algebraic repulsions between charged planes with strongly overlapping electrical double layers, Langmuir 29 (9) (2013) 2859-2870.

[14] T. Markovich, D. Andelman, R. Podgornik, Charge regulation: a generalized boundary condition? EPL 113 (2016) 26004.

[15] F.J.M. Ruiz-Cabello, M. Moazzami-Gudarzi, M. Elzbieciak-Wodka, P. Maroni, C. Labbez, M. Borkovec, G. Trefalt, Long-ranged and soft interactions between charged colloidal particles induced by multivalent coions, Soft Matter 11 (Feb. 2015) 1562-1571.

[16] A.P. Philipse, Remarks on the Donnan condenser in the sedimentation-diffusion equilibrium of charged colloids, Journal of Physics: Condensed Matter 16 (38) (2004) S4051.

[17] A. Philipse, A. Vrij, The Donnan equilibrium: I. On the thermodynamic foundation of the Donnan equation of state, J. Phys. Condens. Matter 23 (19) (2011) 194106.

[18] P.M. Biesheuvel, Evidence for charge regulation in the sedimentation of charged colloids, J. Phys. Condens. Matter 16 (49) (2004) L499.

[19] R.K. Iler, The Chemistry of Silica: Solubility, Polymerization, Colloid and Surface Properties, and Biochemistry, Wiley, 1979.

[20] T.F. Tadros, J. Lyklema, Adsorption of potential-determining ions at the silicaaqueous electrolyte interface and the role of some cations, J. Electroanal. Chem. Interfacial Electrochem. 17 (3) (1968) 267-275.

[21] G.J. Fleer, M.A. Cohen Stuart, J.M.H.M. Scheutjens, T. Cosgrove, B. Vincent, Polymers at Interfaces, Springer Science \& Business Media, 1993.

[22] F.A.M. Leermakers, J.C. Eriksson, J. Lyklema, Fundamentals of Interface and Colloid Science: Soft Colloids, 5 Academic Press, 2005.

[23] J. van Male, Self-consistent-field theory for chain molecules: extensions, computational aspects, and applications, PhD thesis Wageningen University, 2003.

[24] J.M.H.M. Scheutjens, G.J. Fleer, Statistical theory of the adsorption of interacting chain molecules. 1. Partition function, segment density distribution, and adsorption isotherms, J. Phys. Chem. 83 (12) (1979) 1619-1635.

[25] M.R. Böhmer, O.A. Evers, J.M.H.M. Scheutjens, Weak polyelectrolytes between two surfaces: adsorption and stabilization, Macromolecules 23 (8) (1990) 2288-2301. 